Pacific

Journal of

Mathematics

LICHNEROWICZ AND OBATA THEOREMS FOR FOLIATIONS

JEFFREy LeE AND Ken RichaRdson 


\title{
LICHNEROWICZ AND OBATA THEOREMS FOR FOLIATIONS
}

\author{
JefFREY LEE AND Ken RichaRdson
}

The standard Lichnerowicz comparison theorem states that if the Ricci curvature of a closed, Riemannian $n$-manifold $M$ satisfies $\operatorname{Ric}(X, X) \geq a(n-1)|X|^{2}$ for every $X \in T M$ for some fixed $a>0$, then the smallest positive eigenvalue $\lambda$ of the Laplacian satisfies $\lambda \geq a n$. The Obata theorem states that equality occurs if and only if $M$ is isometric to the standard $n$-sphere of constant sectional curvature $a$. In this paper, we prove that if $M$ is a closed Riemannian manifold with a Riemannian foliation of codimension $q$, and if the normal Ricci curvature satisfies $\operatorname{Ric}^{\perp}(X, X) \geq a(q-1)|X|^{2}$ for every $X$ in the normal bundle for some fixed $a>0$, then the smallest eigenvalue $\lambda_{B}$ of the basic Laplacian satisfies $\lambda_{B} \geq a q$. In addition, if equality occurs, then the leaf space is isometric to the space of orbits of a discrete subgroup of $O(q)$ acting on the standard $q$-sphere of constant sectional curvature $a$. We also prove a result about bundle-like metrics on foliations: On any Riemannian foliation with bundle-like metric, there exists a bundle-like metric for which the mean curvature is basic and the basic Laplacian for the new metric is the same as that of the original metric.

\section{Introduction.}

Many researchers have studied relationships between the eigenvalues of the Laplacian and geometric quantities on a Riemannian manifold. For example, the classical Lichnerowicz inequality $([\mathbf{9}])$ states that if the Ricci curvature of an $n$-manifold satisfies

$$
\operatorname{Ric}(X, X) \geq a(n-1)|X|^{2}
$$

for some $a>0$ for every tangent vector $X$, then the first positive eigenvalue of the Laplacian satisfies $\lambda \geq$ an. Obata's Theorem ([12]) states that equality occurs if and only if the manifold is the $n$-sphere with constant sectional curvature $a$.

In this paper we generalize these results to the setting of Riemannian foliations. A Riemannian foliation is a foliation $\mathcal{F}$ on a smooth $n$-manifold $M$ such that the quotient bundle $Q=T M / T \mathcal{F}$ is endowed with a metric 
whose Lie derivative is zero along leaf directions (see [15]). Note that one may always choose a Riemannian metric on $M$ that restricts to the given metric on $Q \cong N \mathcal{F}$; such a metric is called bundle-like. Perhaps the most obvious generalization of the Laplacian would an operator on basic functions (those constant on the leaves) whose local expression is simply the Laplacian on the local quotient. Such an operator would be well-defined in the category of Riemannian foliations but does not conveniently give rise to a self-adjoint operator on any naturally defined Hilbert space of functions on $M$. Therefore, a different approach has become standard, which involves choosing a bundle-like metric on $M$. This new Laplacian, called the basic Laplacian, is essentially self-adjoint on the set of $L^{2}$ basic functions, but it depends on the choice of the bundle-like metric ${ }^{1}$. It is interesting to find situations where this operator will give generalizations to known theorems concerning the ordinary Laplacian. For examples of research in this area, see $[\mathbf{1}],[\mathbf{6}],[\mathbf{7}],[\mathbf{8}],[\mathbf{1 1}],[\mathbf{1 3}]$, and $[\mathbf{1 8}]$.

The basic Laplacian on forms is defined as follows. The set of basic forms, $\Omega_{B}^{*}(M)$, is a subspace of smooth forms $\Omega^{*}(M)$ defined by

$$
\Omega_{B}(M)=\left\{\omega \in \Omega^{*}(M) \mid i(X) \omega=0 \text { and } i(X) d \omega=0 \forall X \in T \mathcal{F}\right\},
$$

where $i(X)$ denotes the interior product with $X$. The exterior derivative maps $\Omega_{B}(M)$ to itself. The basic Laplacian is defined by

$$
\Delta_{B}=\delta_{B} d+d \delta_{B}
$$

where $\delta_{B}$ is the adjoint of $d$ on $L^{2}\left(\Omega_{B}^{*}\right)$. If $\mathcal{F}$ is the foliation by points of $M$, then the basic Laplacian is the ordinary Laplacian. In the more general case, the basic Laplacian and its spectrum provide information about the transverse geometry of $(M, \mathcal{F})$. Since this operator is not a differential operator on the space of all sections of any vector bundle, many of the standard facts about such operators do not easily follow for the basic Laplacian.

For later use, we review the general setup and parts of the proofs of the Weitzenböck formula, the Lichnerowicz inequality, and the Obata theorem in Section 2. In Section 3, we prove the following generalization of the Lichnerowicz inequality. Let $(M, \mathcal{F})$ be a codimension- $q$ Riemannian foliation on a closed, connected Riemannian manifold with a bundle-like metric. Suppose that there exists a positive constant $a$ such that the normal Ricci curvature satisfies $\operatorname{Ric}^{\perp}(X, X) \geq a(q-1)|X|^{2}$ for every $X \in N \mathcal{F}$. Then the smallest nonzero eigenvalue $\lambda_{B}$ of the basic Laplacian on functions satisfies

$$
\lambda_{B} \geq \frac{a q}{1+c_{\kappa}^{2}},
$$

where $c_{\kappa}$ measures the failure of the mean curvature to be parallel along the leaves of the foliation.

\footnotetext{
${ }^{1}$ In [15], Reinhart defines the "basic Laplacian" as the Laplacian on the local quotient rather than the self-adjoint operator that is now commonly called the basic Laplacian.
} 
In Section 4, we show a result about mean curvature and bundle-like metrics that may be of independent interest. We show that if $(M, \mathcal{F})$ is a Riemannian foliation with a bundle-like metric $g$ and corresponding mean curvature form $\kappa$, then there exists a bundle-like metric $\bar{g}$ for $(M, \mathcal{F})$ that has the following properties:

1. It induces the same transverse Riemannian structure as the original metric.

2. The $L^{2}$ metric on basic forms induced from $\bar{g}$ is the same as that of $g$.

3. The basic Laplacian $\overline{\Delta_{B}}$ corresponding to $\bar{g}$ satisfies $\overline{\Delta_{B}}=\Delta_{B}$.

4. The mean curvature form $\bar{\kappa}$ of $(M, \mathcal{F})$ with respect to $\bar{g}$ is a basic form $\left(c_{\bar{\kappa}}=0\right)$.

Hence, (1.1) may always be improved to

$$
\lambda_{B} \geq a q .
$$

We remark that it is surprising that a lower bound can be found for $\lambda_{B}$ that does not depend on mean curvature or volumes of leaves, since the basic Laplacian certainly does.

In Section 5, we consider the case of equality in (1.2), giving a generalization of the Obata theorem to the category of Riemannian foliations. We prove that equality occurs if and only if:

1. The foliation is transversally isometric (see Definition 5.1) to the action of a discrete subgroup of $O(q)$ on the $q$-sphere of constant curvature $a$. Thus, there are at least two closed leaves (the poles).

2. If the mean curvature form is basic, then it is zero (the foliation is minimal).

3. Each level set of the $\lambda_{B}$ eigenfunction is the set of leaves corresponding to a latitude of the $q$-sphere, and the volume $V(r)$ of this level set is the volume of the maximum leaf $L$ times the volume of the latitude.

Finally, we demonstrate the generalized Lichnerowicz and Obata theorems in some examples in Section 6. We demonstrate how these theorems about foliations can be used to prove interesting results about the smallest positive eigenvalue of differential operators on spheres.

Related to this paper is the research in [6], where the researcher proved that $\operatorname{Ric}^{\perp}(X, X) \geq a(q-1)|X|^{2}$ implies that the Riemannian foliation is taut (meaning that there exists a metric for which the leaves are minimal). This implies that the basic projection of the mean curvature form is an exact form. Furthermore, the researcher shows that the transverse diameter $d(M, \mathcal{F})$ of $(M, \mathcal{F})$ satisfies

$$
\begin{aligned}
d(M, \mathcal{F}) & :=\sup _{L_{1}, L_{2} \in \mathcal{F}} \operatorname{dist}\left(L_{1}, L_{2}\right) \\
& \leq \frac{\pi}{\sqrt{a}} .
\end{aligned}
$$


This is a generalization of Myer's Theorem to Riemannian foliations. Also, in [8] the researchers establish geometric upper and lower bounds for the first Dirichlet eigenvalue for the basic Laplacian on a metric tubular neighborhood of a leaf closure, generalizing Cheng's Theorem and other eigenvalue comparison theorems to the category of Riemannian foliations.

\section{The standard Lichnerowicz and Obata theorems.}

We now review some standard results concerning the ordinary Laplacian of a Riemannian manifold, which may be found in $[\mathbf{2}$, pp. 82-84], [5, pp. 113], and [14, pp. 150-165]. Let $M$ be an $n$-dimensional, closed, connected Riemannian manifold. First, recall the ordinary Weitzenböck formula for a form $\omega \in \Omega^{p}(M, E)$, where $E \rightarrow M$ is a Riemannian vector bundle with connection $\nabla$. Let $\nabla^{M}$ denote the Levi-Civita covariant derivative with corresponding curvature $R^{M}$ on $M$. That is,

$$
R^{M}(X, Y)=\left[\nabla_{X}, \nabla_{Y}\right]-\nabla_{[X, Y]} .
$$

Let $\Delta^{E}=d_{\nabla}^{*} d_{\nabla}+d_{\nabla} d_{\nabla}^{*}$, where $d_{\nabla}: \Omega^{p}(M, E) \rightarrow \Omega^{p+1}(M, E)$ is the exterior covariant derivative associated to $\nabla$, and let $R^{E}$ be the curvature of the vector bundle (similarly defined). We now define the map $R(X, Y)$ : $\Omega^{p}(M, E) \rightarrow \Omega^{p}(M, E)$ by

$$
\begin{aligned}
(R(X, Y) \alpha)\left(Z_{1}, \ldots, Z_{p}\right)= & R^{E}(X, Y)\left(\alpha\left(Z_{1}, \ldots, Z_{p}\right)\right) \\
& -\sum_{i=1}^{p} \alpha\left(Z_{1}, \ldots, R^{M}(X, Y) Z_{i}, \ldots, Z_{p}\right) .
\end{aligned}
$$

Note that $d_{\nabla}^{2}=R \wedge$. Let $\Delta=\delta d+d \delta$ denote the Laplacian on $\Omega^{*}(M)$. We endow $\Omega^{p}(M, E)$ and $\Omega^{p}\left(M, E \otimes T^{*} M\right)$ with the natural pointwise inner products, and both pointwise inner products will be denoted by $\langle\cdot, \cdot\rangle$. Then the standard Weitzenböck formulas are

$$
\begin{aligned}
\Delta^{E} \omega & =-\operatorname{tr}\left(\nabla^{2} \omega\right)+\rho(\omega), \\
\frac{1}{2} \Delta\left(|\omega|^{2}\right) & =\left\langle\Delta^{E} \omega, \omega\right\rangle-|\nabla \omega|^{2}-\langle\rho(\omega), \omega\rangle,
\end{aligned}
$$

where $\rho: \Omega^{p}(M, E) \rightarrow \Omega^{p}(M, E)$ is the generalized Ricci curvature operator defined by

$$
\begin{aligned}
& \rho(\omega)\left(Z_{1}, \ldots, Z_{p}\right) \\
& = \begin{cases}0 & \text { if } p=0 \\
\sum_{k, s}\left(R\left(E_{s}, Z_{k}\right) \omega\right)\left(Z_{1}, \ldots, Z_{k-1}, E_{s}, Z_{k+1}, \ldots, Z_{p}\right) & \text { if } p>0\end{cases}
\end{aligned}
$$

where $\left\{E_{1}, \ldots, E_{n}\right\}$ is an orthonormal basis of $T_{x} M$. The Ricci tensor

$$
\operatorname{Ric}(X, Y)=\operatorname{tr}\left(Z \longmapsto R^{M}(Z, X) Y\right)=\sum_{s}\left\langle R^{M}\left(E_{s}, X\right) Y, E_{s}\right\rangle
$$


on $M$ may be thought of as an element of $\Omega^{1}(M, T M)$ by $\operatorname{Ric}(X)=$ $\operatorname{Ric}(X, *)^{\#}$ or $\operatorname{Ric}(X, Y)=\langle\operatorname{Ric}(X), Y\rangle$. This tensor is the restriction of $\rho$ to $\Omega^{1}(M)$ by $(\rho(\omega))^{\#}=\operatorname{Ric}\left(\omega^{\#}\right)$.

Applying Formula $(2.1)$ to $\Omega^{0}(M, E)$, we obtain

$$
\frac{1}{2} \Delta\left(|s|^{2}\right)=\left\langle\Delta^{E} s, s\right\rangle-|\nabla s|^{2} .
$$

Applying Formula $(2.1)$ to $\Omega^{1}(M, E)$, we obtain

$$
\begin{aligned}
\frac{1}{2} \Delta\left(|\alpha|^{2}\right)= & \left\langle\Delta^{E} \alpha, \alpha\right\rangle-|\nabla \alpha|^{2}-\sum_{j, k}\left\langle R^{E}\left(E_{j}, E_{k}\right) \alpha\left(E_{j}\right), \alpha\left(E_{k}\right)\right\rangle \\
& -\sum_{i}\left\langle\alpha\left(\operatorname{Ric}\left(E_{i}\right)\right), \alpha\left(E_{i}\right)\right\rangle .
\end{aligned}
$$

Given a smooth function $f: M \rightarrow \mathbb{R}$, then $d f \in \Omega^{1}(M, \mathbb{R})$, which implies that

$$
\begin{aligned}
\frac{1}{2} \Delta\left(|d f|^{2}\right) & =\langle\Delta d f, d f\rangle-|\nabla d f|^{2}-\sum_{i} d f\left(\operatorname{Ric}\left(E_{i}\right)\right) \cdot d f\left(E_{i}\right) \\
& =\langle d \delta d f, d f\rangle-|\nabla d f|^{2}-\sum_{i}\left\langle\operatorname{Ric}\left(E_{i}\right), E_{j}\right\rangle E_{j}(f) E_{i}(f) \\
& =\langle d \delta d f, d f\rangle-|\nabla d f|^{2}-\operatorname{Ric}(\nabla f, \nabla f) \\
& =\langle d \delta d f, d f\rangle-|\operatorname{Hess}(f)|^{2}-\operatorname{Ric}(\nabla f, \nabla f) .
\end{aligned}
$$

Here, if $T$ is a linear transformation on an inner product space, $|T|=$ $\sqrt{\operatorname{tr}\left(T^{*} T\right)}$ is the standard norm of $T$. If we let $f$ be a normalized eigenfunction of $\Delta$ with eigenvalue $\lambda$, we have that

$$
\begin{aligned}
\frac{1}{2} \Delta\left(|d f|^{2}\right) & =\lambda\langle d f, d f\rangle-|\operatorname{Hess}(f)|^{2}-\operatorname{Ric}(\nabla f, \nabla f) . \\
& \leq \lambda\langle d f, d f\rangle-\frac{|\operatorname{tr}(\operatorname{Hess}(f))|^{2}}{n}-\operatorname{Ric}(\nabla f, \nabla f) \\
& =\lambda\langle d f, d f\rangle-\frac{|\Delta f|^{2}}{n}-\operatorname{Ric}(\nabla f, \nabla f),
\end{aligned}
$$

where we have used the fact any linear transformation $L: H \rightarrow H$ on any $n$-dimensional inner product space $H$ satisfies $|L|^{2} \geq \frac{|\operatorname{tr}(L)|^{2}}{n}$, with equality if and only if $L$ is a scalar multiple of the identity. Suppose that for some $a>0$, Ric $(X, X) \geq a(n-1)|X|^{2}$ for every $X \in T M$. Integrating the above inequality over $M$, we have that

$$
0 \leq \lambda^{2}-\frac{\lambda^{2}}{n}-\int_{M}\left(a(n-1)|\nabla f|^{2}\right) d V=\frac{n-1}{n} \lambda^{2}-a(n-1) \lambda,
$$


which implies that

$$
\lambda \geq a n
$$

This inequality above is due to Lichnerowicz. Obata proved that equality in the formula above implies that $M$ is isometric to the $n$-sphere of constant sectional curvature $a$. See $[\mathbf{9}]$ and $[\mathbf{1 2}]$ for the original proofs.

\section{Riemannian foliations and the generalized Lichnerowicz inequality.}

Now, consider a codimension- $q$ Riemannian foliation $(M, \mathcal{F})$. Let $f: M \rightarrow \mathbb{R}$ be a smooth basic function. By (2.2), we have that

$$
\begin{aligned}
\frac{1}{2} \Delta\left(|d f|^{2}\right) & =\langle d \delta d f, d f\rangle-|\nabla d f|^{2}-\operatorname{Ric}(\nabla f, \nabla f) \\
& =\langle d \delta d f, d f\rangle-\left|\operatorname{Hess}^{\perp}(f)\right|^{2}-\operatorname{Ric}^{\perp}(\nabla f, \nabla f),
\end{aligned}
$$

where $\mathrm{Ric}^{\perp}$ is the Ricci tensor restricted to the normal bundle and Hess ${ }^{\perp}$ is the Hessian restricted to the normal bundle. We have used the fact that $d f$ is a basic form, implying that its covariant derivative $\nabla d f(X, Y)$ is zero if either $X \in T \mathcal{F}$ or $Y \in T \mathcal{F}$. Note that because $M$ is Riemannian, the normal Ricci curvature tensor, when applied to basic vector fields $X, Y \in \Gamma N \mathcal{F}$, satisfies

$$
\begin{aligned}
\operatorname{Ric}(X, Y) & =\operatorname{tr}\left(Z \longmapsto R^{M}(Z, X) Y\right)=\sum_{s}\left\langle R^{M}\left(E_{s}, X\right) Y, E_{s}\right\rangle \\
& =\sum_{E_{s} \in N \mathcal{F}}\left\langle R^{M}\left(E_{s}, X\right) Y, E_{s}\right\rangle=\operatorname{tr}^{\perp}\left(Z \longmapsto R^{M}(Z, X) Y\right) \\
& =: \operatorname{Ric}^{\perp}(X, Y) .
\end{aligned}
$$

If we have that the normal Ricci curvature satisfies

$$
\operatorname{Ric}^{\perp}(X, X) \geq a(q-1)|X|^{2},
$$

then

$$
\begin{aligned}
\frac{1}{2} \Delta\left(|d f|^{2}\right) & \leq\langle d \delta d f, d f\rangle-\frac{\left(\operatorname{tr}\left(\operatorname{Hess}^{\perp}(f)\right)\right)^{2}}{q}-a(q-1)|\nabla f|^{2} \\
& =\langle d \delta d f, d f\rangle-\frac{(\operatorname{tr}(\operatorname{Hess}(f)))^{2}}{q}-a(q-1)|\nabla f|^{2} \\
& =\langle d \delta d f, d f\rangle-\frac{|\Delta f|^{2}}{q}-a(q-1)|\nabla f|^{2} .
\end{aligned}
$$


Integrating over $M$, we have that

$$
\begin{aligned}
0 & \leq \frac{q-1}{q} \int_{M}\langle d \delta d f, d f\rangle-a(q-1) \int_{M}\langle\Delta f, f\rangle \\
& =\frac{q-1}{q} \int_{M}(\Delta f)^{2}-a(q-1) \int_{M}\langle\Delta f, f\rangle .
\end{aligned}
$$

Next, recall the following formula from [13] for the basic Laplacian of a basic function $g$ :

$$
\Delta_{B} g=\Delta g+\langle d g,(P \kappa-\kappa)\rangle
$$

where $\kappa$ is the mean curvature form of the foliation and $P: \Omega^{*}(M) \rightarrow$ $\Omega_{B}^{*}(M)$ is the $L^{2}$-orthogonal projection onto basic forms. Also, $\int_{M} g=$ $\int_{M} P g$ for any function $g$, and $P\langle\alpha, \beta\rangle=\langle P \alpha, \beta\rangle$ if $\beta$ is a basic form and $\alpha$ is any form (see $[\mathbf{1 3}]$ for details). Let the leafwise deviation $c_{\kappa}$ of the mean curvature of the foliation be

$$
c_{\kappa}=\max _{x \in M}\|P \kappa-\kappa\|_{x}=\max _{x \in M}\|P H-H\|_{x},
$$

where $H$ is the mean curvature vector field. Observe that $c_{\kappa}$ measures the failure for the mean curvature vector field to be parallel along the leaves that is, the failure for the mean curvature form to be a basic form.

Proposition 3.1 (Generalized Lichnerowicz Inequality-First Version). Let $(M, \mathcal{F})$ be a codimension- $q$ Riemannian foliation on a closed, connected Riemannian manifold with leafwise mean curvature deviation $c_{\kappa}$. Suppose that there exists a positive constant a such that the normal Ricci curvature satisfies $\operatorname{Ric}^{\perp}(X, X) \geq a(q-1)|X|^{2}$ for every $X \in N \mathcal{F}$. Then the smallest nonzero eigenvalue $\lambda_{B}$ of the basic Laplacian satisfies

$$
\lambda_{B} \geq \frac{a q}{1+c_{\kappa}^{2}}
$$


Proof. Let $f$ be a normalized eigenfunction of the basic Laplacian with eigenvalue $\lambda_{B}>0$. By (3.2) and the remarks above,

$$
\begin{aligned}
0 \leq & \frac{q-1}{q} \int_{M}(\Delta f)^{2}-a(q-1) \int_{M} f \Delta f \\
= & \frac{q-1}{q} \int_{M}\left(\Delta_{B} f-\langle d f,(P \kappa-\kappa)\rangle\right)^{2}-a(q-1) \int_{M} P(f \Delta f) \\
= & \frac{q-1}{q} \int_{M}\left(\lambda_{B}^{2} f^{2}-2 \lambda_{B} f\langle d f,(P \kappa-\kappa)\rangle+\langle d f,(P \kappa-\kappa)\rangle^{2}\right) \\
& -a(q-1) \int_{M} f P \Delta f \\
\leq & \frac{q-1}{q}\left(\lambda_{B}^{2}-2 \lambda_{B} \int_{M} P(f\langle d f,(P \kappa-\kappa)\rangle)+c_{\kappa}^{2} \int_{M}|d f|^{2}\right) \\
& -a(q-1) \int_{M} f \Delta_{B} f \\
= & \frac{q-1}{q}\left(\lambda_{B}^{2}-2 \lambda_{B} \int_{M} f\langle d f, P(P \kappa-\kappa)\rangle+c_{\kappa}^{2} \lambda_{B}^{2}\right)-a(q-1) \lambda_{B} \\
= & \frac{q-1}{q}\left(1+c_{\kappa}^{2}\right) \lambda_{B}^{2}-a(q-1) \lambda_{B},
\end{aligned}
$$

and the proposition follows.

We next consider the constant $c_{\kappa}$. Obviously, if the mean curvature of the foliation is basic, then this constant vanishes. In the next section, we show that the bundle-like metric can be modified so that the mean curvature is basic and so that the basic Laplacian corresponding to the new metric is the same operator as the original basic Laplacian. As a corollary, we get an improvement to the proposition above.

\section{Mean curvature, the basic Laplacian, and bundle-like metrics.}

In [4], the researcher showed that given a Riemannian foliation $(M, \mathcal{F})$ with bundle-like metric $g$, there exists another bundle-like metric $g^{\prime}$ that induces the same metric on $T M / T \mathcal{F} \cong N^{\prime} \mathcal{F}$ and whose mean curvature form $\kappa^{\prime}$ is basic. Moreover, as explained in [1] and [4], a given bundle-like metric on a Riemannian foliation may be deformed - by multiplying the metric along the leaves by a positive function or by making a different choice of the subbundle $N \mathcal{F}$ - to get any possible mean curvature form. In fact, the mean curvature only depends the normal bundle $N \mathcal{F}$ and the leafwise metric, so that it would not change if the transverse Riemannian structure were modified. Let $(\cdot, \cdot)$ and $(\cdot, \cdot)^{\prime}$ denote the pointwise inner products of forms for any two bundle-like metrics $g$ and $g^{\prime}$ that induce identical metrics on $T M / T \mathcal{F}$, and let $\langle\cdot, \cdot\rangle$ and $\langle\cdot, \cdot\rangle^{\prime}$ be the corresponding $L^{2}$ inner products 
of forms. In what follows, let $p$ denote the dimension of the foliation and $q=n-p$ the codimension. For any two basic forms $\alpha$ and $\beta$,

$$
\begin{aligned}
\langle\alpha, \beta\rangle^{\prime} & =\int_{M}(\alpha, \beta)^{\prime} \chi^{\prime} \wedge \nu^{\prime} \\
& =\int_{M}(\alpha, \beta) e^{\phi} \chi \wedge \nu,
\end{aligned}
$$

where, for example, $\chi=E_{1}^{*} \wedge \cdots \wedge E_{p}^{*}$ and $\nu=E_{p+1}^{*} \wedge \cdots \wedge E_{p+q}^{*}$ are the characteristic form and transversal volume form of the foliation (see [18]; $\left\{E_{1}, \ldots, E_{p}, E_{p+1}, \ldots, E_{p+q}\right\}$ is a local adapted frame field), and $e^{\phi}$ is the positive function such that $\chi^{\prime} \wedge \nu=e^{\phi} \chi \wedge \nu$. Observe that since $T \mathcal{F}$ is involutive and the induced transverse metrics are the same, we have that the induced metrics on the bundle $(T M / T \mathcal{F})^{*}$ are the same and thus that $\nu^{\prime}=\nu$.

The following result concerns the basic Laplacians of $g$ and $g^{\prime}$.

Proposition 4.1. Let $g$ and $g^{\prime}$ be any two bundle-like metrics on the Riemannian foliation $(M, \mathcal{F})$ that induce identical metrics on $T M / T \mathcal{F}$, and let $\delta_{B}$ and $\delta_{B}^{\prime}$ be the adjoints of $d_{B}$ with respect to the $L^{2}$ metrics on forms induced from $g$ and $g^{\prime}$, respectively. Then there exists a positive, smooth, basic function $\psi$ such that

$$
\delta_{B}^{\prime} \beta=\frac{1}{\psi} \delta_{B}(\psi \beta)
$$

for any smooth basic form $\beta$.

Proof. Given any smooth basic forms $\alpha$ and $\beta$, by (4.1) we have that

$$
\begin{aligned}
\langle d \alpha, \beta\rangle^{\prime} & =\int_{M}(d \alpha, \beta) e^{\phi} \chi \wedge \nu \\
& =\int_{M}(d \alpha, \beta) P\left(e^{\phi}\right) \chi \wedge \nu,
\end{aligned}
$$

where $P: L^{2}\left(\Omega^{*}(M)\right) \longrightarrow L^{2}\left(\Omega_{B}^{*}(M)\right)$ is the orthogonal projection with respect to the original metric $g$. We have use the following facts for Riemannian foliations (see $[\mathbf{1 3}]$ ):

1. $(d \alpha, \beta)$ is a basic function,

2. $\int f d V=\int P(f) d V$ for any $L^{2}$ function $f$, and

3. $P(\eta \omega)=\eta P(\omega)$ if $\eta$ is any $L^{2}$ basic function and $\omega$ is any $L^{2}$ function.

Let $\psi=P\left(e^{\phi}\right)$, which by the results of [13] is a smooth, positive, basic function. We then have that

$$
\begin{aligned}
\langle d \alpha, \beta\rangle^{\prime} & =\int_{M}(d \alpha, \psi \beta) \chi \wedge \nu \\
& =\int_{M}\left(\alpha, \frac{1}{\psi} \delta_{B}(\psi \beta)\right) \psi \chi \wedge \nu=\left\langle\alpha, \frac{1}{\psi} \delta_{B}(\psi \beta)\right\rangle^{\prime} .
\end{aligned}
$$


Corollary 4.2. Let $(M, \mathcal{F})$ be a Riemannian foliation with a bundle-like metric $g$ and corresponding mean curvature form $\kappa$. Let $\langle\cdot, \cdot\rangle$ be the associated $L^{2}$ metric on basic forms, and let $\Delta_{B}$ be the basic Laplacian on forms. Then there exists a bundle-like metric $\bar{g}$ for $(M, \mathcal{F})$ that has the following properties:

1. It induces the same transverse Riemannian structure as the original metric.

2. The $L^{2}$ metric $\overline{\langle\cdot, \cdot\rangle}$ on basic forms induced from $\bar{g}$ is the same as $\langle\cdot, \cdot\rangle$.

3. The basic Laplacian $\overline{\Delta_{B}}$ corresponding to $\bar{g}$ satisfies $\overline{\Delta_{B}}=\Delta_{B}$.

4. The mean curvature form $\bar{\kappa}$ of $(M, \mathcal{F})$ with respect to $\bar{g}$ is a basic form.

Proof. By the results of [4], there exists a bundle-like metric $\widetilde{g}$ for $(M, \mathcal{F})$ such that the mean curvature $\widetilde{\kappa}$ is basic and that induces the same metric on $(T M / T \mathcal{F})$ as that from the metric $g$. By Proposition 4.1 and its proof, the new $L^{2}$ metric $\widetilde{\langle\cdot, \cdot\rangle}$ and basic adjoint $\widetilde{\delta}_{B}$ satisfy the following for any smooth basic forms $\alpha, \beta, \gamma$.

$$
\widetilde{\langle\cdot, \cdot\rangle}=\langle\psi \cdot, \cdot\rangle, \widetilde{\delta}_{B} \beta=\frac{1}{\psi} \delta_{B}(\psi \beta)
$$

for some fixed, smooth, positive, basic function $\psi$. Next, we modify the leafwise metric from $\widetilde{g}$ by multiplying it by $\psi^{-2 / p}$ to get the new metric $\bar{g}$. Observe that $\bar{\chi} \wedge \nu=\frac{1}{\psi} \widetilde{\chi} \wedge \nu$, so that as in the proof of Proposition 4.1, we have that

$$
\begin{aligned}
& \overline{\langle\cdot, \cdot\rangle}=\widetilde{\left\langle\frac{1}{\psi} \cdot, \cdot\right\rangle}=\langle\cdot, \cdot\rangle, \text { and } \\
& \bar{\delta}_{B} \beta=\psi \widetilde{\delta}_{B}\left(\frac{1}{\psi} \beta\right)=\psi \frac{1}{\psi} \delta_{B}\left(\psi \frac{1}{\psi} \beta\right)=\delta_{B} \beta .
\end{aligned}
$$

Thus, $\bar{g}$ induces the same transverse Riemannian structure, the same $L^{2}$ metric on forms, and the same basic adjoint of $d_{B}$ as the original metric $g$. Therefore, the basic Laplacian on basic forms that in induced from $\bar{g}$ is the same operator as that coming from the original metric.

Next, by Rummler's formula $d \chi=-\kappa \wedge \chi+\varphi_{0}$ (see [16], [18], [1], [13]), it is easy to see that the mean curvature of $(M, \mathcal{F})$ with respect to the metric $\bar{g}$ satisfies $\bar{\kappa}=\widetilde{\kappa}+\frac{1}{\psi} d \psi$, which is the sum of two basic one-forms.

In [13], the researchers showed that the basic Laplacian on functions is the restriction of the ordinary Laplacian on functions if and only if the mean curvature of the Riemannian foliation is basic. We then have the following:

Corollary 4.3. Let $(M, \mathcal{F})$ be a Riemannian foliation with a bundle-like metric $g$, and let $\Delta_{B}$ be the basic Laplacian on functions. Then there exists a bundle-like metric $\bar{g}$ such that $\Delta_{B}$ is the restriction of the ordinary Laplacian 
$\bar{\Delta}$ corresponding to $\bar{g}$ to basic forms. Therefore, the spectrum of $\Delta_{B}$ is contained in the spectrum of $\bar{\Delta}$, and the eigenspaces of $\Delta_{B}$ are subsets of eigenspaces of $\bar{\Delta}$.

We now combine Proposition 3.1 and Corollary 4.2 to obtain the following result.

Theorem 4.4 (Generalized Lichnerowicz Inequality). Let $(M, \mathcal{F})$ be a codimension-q Riemannian foliation on a closed, connected Riemannian manifold. Suppose that there exists a positive constant a such that the normal Ricci curvature satisfies $\operatorname{Ric}^{\perp}(X, X) \geq a(q-1)|X|^{2}$ for every $X \in N \mathcal{F}$. Then the smallest nonzero eigenvalue $\lambda_{B}$ of the basic Laplacian satisfies

$$
\lambda_{B} \geq a q .
$$

Observe that the trivial foliation by points yields the standard Lichnerowicz Theorem $\lambda \geq$ an. Note that the mean curvature and volumes of the leaves do have an effect on the basic Laplacian and its eigenvalues, so it is somewhat surprising that a condition on the transverse geometry alone is enough to give a lower bound for the first positive eigenvalue of the basic Laplacian.

\section{The generalized Obata theorem for Riemannian foliations.}

Suppose we have the case of equality in Theorem 4.4. We may assume that the mean curvature is basic, because of Corollary 4.2. In light of (3.3), the basic Laplacian on functions is the restriction of the ordinary Laplacian $\Delta$ to basic functions, so that $\lambda_{B}$ is an eigenvalue of $\Delta$ as well.

Next, we follow the line of reasoning in $[\mathbf{2}$, p. 84]. We consider the proof of Proposition 3.1. Equality implies that $\frac{\left(\operatorname{tr}\left(\operatorname{Hess}^{\perp}(f)\right)\right)^{2}}{q}=\left|\operatorname{Hess}^{\perp}(f)\right|^{2}$, so that there exists a smooth basic function $\phi$ such that $\operatorname{Hess}^{\perp}(f)=\phi I_{N \mathcal{F}}$ at each point of $M$. Then

$$
a q f=\lambda_{B} f=\Delta_{B} f=\Delta f=-\operatorname{tr}(\operatorname{Hess}(f))=-\operatorname{tr}\left(\operatorname{Hess}^{\perp}(f)\right)=-q \phi,
$$

so that

$$
\operatorname{Hess}(f)=-(a f) P_{N \mathcal{F}},
$$

where $P_{N \mathcal{F}}: T M \rightarrow N \mathcal{F}$ denotes the obvious projection. For any unit speed geodesic $\gamma:(\alpha, \beta) \rightarrow M$ that is normal to the foliation,

$$
\begin{aligned}
-a(f \circ \gamma) & =-a(f \circ \gamma)\left|\gamma^{\prime}\right|^{2}=\left\langle\operatorname{Hess}(f)\left(\gamma^{\prime}\right), \gamma^{\prime}\right\rangle \\
& =\left\langle\nabla_{\gamma^{\prime}} \nabla f, \gamma^{\prime}\right\rangle \\
& =\left\langle\nabla f, \gamma^{\prime}\right\rangle^{\prime}-\left\langle\nabla f, \nabla_{\gamma^{\prime}} \gamma^{\prime}\right\rangle \\
& =(f \circ \gamma)^{\prime \prime},
\end{aligned}
$$


so that

$$
(f \circ \gamma)(t)=A \cos \sqrt{a} t+B \sin \sqrt{a} t .
$$

In particular, if $p \in M$ is either a global maximum or minimum of $f$ on $M$, then

$$
f\left(\gamma_{\xi}(t)\right)=f(p) \cos \sqrt{a} t
$$

for any $\xi \in N_{p} \mathcal{F}$. This implies that the normal exponential map $\exp _{p}^{\perp}$ : $N_{p} \mathcal{F} \rightarrow M$ is injective on the ball $B_{\pi / \sqrt{a}}(p)$, so that the transverse diameter

$$
\begin{aligned}
d(M, \mathcal{F}) & :=\sup _{L_{1}, L_{2} \in \mathcal{F}} \operatorname{dist}\left(L_{1}, L_{2}\right) \\
& \geq \pi / \sqrt{a} .
\end{aligned}
$$

Suppose a global maximum is achieved at that $p \in M$; the formula above implies that the maximum is isolated to the (closed) leaf containing $p$; similarly, global minima are isolated to closed leaves. Choose a closed leaf $L$ corresponding to a global maximum and a closed leaf $L^{\prime}$ corresponding to a global minimum, and connect them by a minimal, unit-speed geodesic $\gamma$ that is therefore normal to the foliation. By applying (5.1) to $\gamma$, we must have that the maximum and minimum values of $f$ are opposites. Assuming $\gamma(0)=p \in L$, we have that the normal exponential map $\exp _{p}^{\perp}$ maps the sphere of radius $\pi / \sqrt{a}$ to $L^{\prime}$, because this global minimum must be isolated. We observe that because the distance between a point of one leaf to another leaf of a Riemannian foliation is independent of the original point chosen, the above discussion is independent of $p \in L$ (the fact that this is true for nearby leaves is standard; see [8] for the more general result). Because the normal exponential map is surjective (see, for example, [8]), $\bigcup_{p \in L} \exp _{p}^{\perp}\left(\overline{B_{\pi / \sqrt{a}}(p)}\right)=M$. Because the distance from $L$ to the cut locus is $\pi / \sqrt{a}$, the balls $\exp _{p}^{\perp}\left(B_{\pi / \sqrt{a}}(p)\right)$ are disjoint.

Recall that the holonomy group of a closed leaf of a Riemannian foliation is isomorphic to a discrete subgroup of $O(q)$, and its action on normal balls is determined by its action on the normal space at a point of the leaf (see $[\mathbf{1 0}]$ ). We conclude that the space of leaves is homeomorphic to the quotient of a topological $q$-dimensional sphere $S$ by a discrete subgroup $G$ of $O(q)$ (thought of as acting on $N_{p} \mathcal{F}$ or the tangent space to a pole of the sphere), since the leaf space is $\overline{B_{\pi / \sqrt{a}}(p)} / S_{\pi / \sqrt{a}}(p)$ modulo holonomy at $p$.

We now put a metric on the $S$ using the metric on the normal bundle of the foliation. Choose a sufficiently small neighborhood $U$ of $p$ so that the foliation restricted to $\bigcup_{p \in U} B_{\frac{\pi}{\sqrt{a}}}(p)$ is a product. The local quotient manifold can be identified with $B_{\frac{\pi}{\sqrt{a}}}(p)$ via the normal exponential map, and the metric on the normal bundle induces a Riemannian metric on the local quotient. By the condition $\operatorname{Ric}^{\perp}(X, X) \geq a(q-1)|X|^{2}$ and by formula 
(5.1), the Ricci curvature $\operatorname{Ric}^{S}$ of the induced metric on $B_{\frac{\pi}{2 \sqrt{a}}}(p)$ satisfies $\operatorname{Ric}^{S}(X, X) \geq a(q-1)|X|^{2}$ for every $X \in T B_{\frac{\pi}{\sqrt{a}}}(p)$. Letting $p$ be the global minimum of $f$, we may go through the same argument to obtain a metric on $S$ with the property that $\operatorname{Ric}^{S}(X, X) \geq a(q-1)|X|^{2}$ for every $X \in T S$. We conclude that the space of leaves is isometric (as a metric space) to the quotient of a topological $q$-dimensional sphere $S$ by a discrete subgroup $G$ of $O(q)$ that acts by isometries.

Even more is true. First, we make the following definition.

Definition 5.1. Let $G$ be a discrete group. We say that a Riemannian foliation $(M, \mathcal{F})$ is transversally isometric to the isometric action of $G$ on a Riemannian manifold $W$ if there exists a smooth, surjective map $\phi: M \rightarrow W$ such that:

1. The function $\phi$ induces a homeomorphism between the leaf space $M / \mathcal{F}$ and the orbit space $W / G$.

2. For each $x \in M$, the push forward $\phi_{*}$ restricts to an isometry $\phi_{*}$ : $N_{x} \mathcal{F} \rightarrow T_{\phi(x)} W$, where $N \mathcal{F}$ is the normal bundle of the foliation and $T W$ is the tangent bundle of $W$.

Remark 5.1. The definition above is an example of an isometric equivalence between two pseudogroups of local isometries acting on smooth manifolds. In this case, the holonomy pseudogroup of the foliation is isometrically equivalent to the pseudogroup generated by the group action. See [17] for a discussion.

Theorem 5.1 (Generalized Obata Theorem). Let $(M, \mathcal{F})$ be a codimension-q Riemannian foliation on a closed, connected Riemannian manifold. Suppose that there exists a positive constant a such that the normal Ricci curvature satisfies $\operatorname{Ric}^{\perp}(X, X) \geq a(q-1)|X|^{2}$ for every $X \in N \mathcal{F}$. We have equality in Theorem $4.4\left(\lambda_{B}=a q\right)$ if and only if:

1. $(M, \mathcal{F})$ is transversally isometric to the action of a discrete subgroup of $O(q)$ acting on the q-sphere of constant curvature $a$. Thus, there are at least two closed leaves (the poles).

2. If we choose the metric on $M$ so that the mean curvature form is basic (Corollary 4.2), then the mean curvature of the foliation is zero (the foliation is minimal).

3. Each level set of the $\lambda_{B}$ eigenfunction is the set of leaves corresponding to a latitude of the q-sphere, and the volume $V(r)$ of this level set is the volume of the maximum leaf $L$ times the volume of the latitude. Explicitly, if $r$ denotes the distance from the level set to $L$, then

$$
V(r)=\operatorname{Vol}\left(S^{q-1}(1)\right) \operatorname{Vol}(L)\left(\frac{\sin (\sqrt{a} r)}{\sqrt{a}}\right)^{q-1} .
$$


Proof. Suppose that we have equality. In the above discussion, we showed that the space of leaves is isometric (as a metric space) to the quotient of a topological $q$-dimensional sphere $S$ by a discrete subgroup $G$ of $O(q)$ that acts by isometries. For (5.1), we transplant the eigenfunction $f$ of $\Delta_{B}$ on $M$ to a function $\bar{f}$ on $S$ via the normal exponential map, and we identify the maximum "pole" with the point $p$. Let $\sqrt{\mathbf{g}}(r, \xi)$ be the function defined by the following formula for the volume form in geodesic polar coordinates $(r, \xi)$ around $p$ :

$$
d V(r, \xi)=\sqrt{\mathbf{g}}(r, \xi) d r d \nu_{\xi},
$$

where $d \nu_{\xi}$ is the volume form of the unit sphere in $T_{p} S$. Note that $\sqrt{\mathbf{g}}(0, \xi)=$ 1 and $\sqrt{\mathbf{g}}(r, \xi)>0$ for $0 \leq r<\frac{\pi}{\sqrt{a}}$. Let

$$
\Theta(r)=\Theta(r, \xi)=\left(\frac{\sin (\sqrt{a} r)}{\sqrt{a}}\right)^{q-1}
$$

denote the function analogous to $\sqrt{\mathbf{g}}(r, \xi)$ for a ball in a $q$-dimensional sphere of constant curvature $a$. By Bishop's Comparison Theorem (see [2, pp. 71-73]),

$$
\begin{aligned}
\frac{\partial}{\partial r}\left(\frac{\sqrt{\mathbf{g}}(r, \xi)}{\Theta(r)}\right) & \leq 0, \text { or } \\
\frac{\partial}{\partial r}(\log \sqrt{\mathbf{g}}(r, \xi)) & \leq \frac{\partial}{\partial r}(\log \Theta(r))=(q-1) \sqrt{a} \cot \sqrt{a} r
\end{aligned}
$$

and $\sqrt{\mathbf{g}}(r, \xi) \leq \Theta(r)$ for $0<r<\frac{\pi}{\sqrt{a}}$.

The Laplacian $\Delta^{S}$ in geodesic polar coordinates $(r, \xi)$ around $p \in S$ acting on functions of $r$ alone is given by the formula

$$
\Delta^{S} \psi(r)=-\frac{d^{2} \psi}{d r^{2}}(r)-\left(\frac{d}{d r} \log (\sqrt{\mathbf{g}}(r, \xi))\right) \frac{d \psi}{d r}(r) .
$$

Dividing by a constant if necessary, we consider the eigenfunction $\psi(r)=$ $\bar{f}(r)=\cos \sqrt{a} r$. Noting that $\frac{d \bar{f}}{d r}(r) \leq 0$, we obtain

$$
\begin{aligned}
\Delta^{S} \bar{f}(r) & =\Delta^{S} \cos \sqrt{a} r=-\frac{d^{2} \bar{f}}{d r^{2}}(r)-\left(\frac{d}{d r} \log (\sqrt{\mathbf{g}}(r, \xi))\right) \frac{d \bar{f}}{d r}(r) \\
& \leq-\frac{d^{2} \bar{f}}{d r^{2}}(r)-((q-1) \sqrt{a} \cot \sqrt{a} r) \frac{d \bar{f}}{d r}(r) \\
& =a q \bar{f}(r) .
\end{aligned}
$$

Since $\bar{f}(r)>0$ for $0 \leq r<\frac{\pi}{2 \sqrt{a}}$ and $\bar{f}\left(\frac{\pi}{2 \sqrt{a}}\right)=0$, we have that the smallest Dirichlet eigenvalue $\lambda_{1}$ for the hemisphere $0 \leq r \leq \frac{\pi}{2 \sqrt{a}}$ satisfies $\lambda_{1} \leq a q$. Repeating the argument for $-\bar{f}$ centered at the opposite pole, we get that 
the smallest Dirichlet eigenvalue $\lambda_{1}^{\prime}$ for the complementary hemisphere satisfies $\lambda_{1}^{\prime} \leq a q$. Letting $\lambda(S)$ denote the smallest positive eigenvalue of the Laplacian on $S$, the Rayleigh min-max method gives

$$
\lambda(S) \leq \max \left\{\lambda_{1}, \lambda_{1}^{\prime}\right\} \leq a q .
$$

On the other hand, the standard Obata Theorem implies that $\lambda(S) \geq a q$, so that $\lambda(S)=a q$. The case of equality in the standard Obata Theorem then implies that $S$ is isometric to the $q$-sphere of constant curvature $a$. Moreover, $\sqrt{\mathbf{g}}(r, \xi)=\Theta(r)$.

To prove (5.1), observe that the induced Laplacian on functions in the local quotient manifold in a Riemannian foliation is the operator

$$
\Delta^{S}=-\bar{*} d \bar{*} d,
$$

where $\bar{*}$ is the Hodge star operator in the local quotient and is discussed in [18]; the operator $\Delta^{S}$ is not necessarily self-adjoint as an operator on basic functions on $M$. The basic Laplacian (in the case of basic mean curvature) satisfies

$$
\Delta_{B} g=-\bar{*} d \bar{*} d g+\langle d g, \kappa\rangle=-\bar{*} d \bar{*} d g+\kappa^{\#}(g)
$$

for any basic function $g$ (see [13]). Note that $\kappa^{\#}$ is the (basic) mean curvature vector field. Thus, $\Delta^{S}$ coincides with $\Delta_{B}$ if and only if the basic projection of the mean curvature of the foliation is zero. In our case we only have that

$$
\Delta_{B} f=\Delta^{S} \bar{f}=a q f,
$$

so that $\kappa^{\#}(f)=0$. Thus $\kappa^{\#}$ is tangent to the level sets of the first basic eigenfunction.

By the results in [6], the lower bound on normal Ricci curvature implies that the foliation is taut, so that the basic mean curvature form is an exact form, so that $\kappa=d h$ for some basic function $h$. Observe that we may connect the maximal leaf $L$ to any other leaf $L^{\prime}$ by a normal geodesic $\gamma$ that is orthogonal to the level sets of $f$. Integrating $\nabla h$ along $\gamma$ yields zero because $\kappa^{\#}=\nabla h$ is tangent to the level sets of $f$. Therefore, we conclude that $h$ is a constant function, so that $\kappa=0$. This proves (5.1).

We now prove (5.1). Observe that since the mean curvature form is basic, the basic Laplacian is the restriction of the ordinary Laplacian, as we mentioned previously. We have that

$$
\Delta f=a q f
$$

and $f$ is a function of $r$ alone, where $r$ is the distance from the maximum leaf $L$. Writing the Laplacian acting on functions of $r$ alone, we have that

$$
\Delta f(r)=\delta d f(r)=P_{r} \delta d f(r)=\left(-\frac{\partial^{2}}{\partial r^{2}}-\frac{\partial}{\partial r}(\log V(r)) \frac{\partial}{\partial r}\right) f(r),
$$


where $P_{r}$ is the $L^{2}$ projection onto functions of $r$ alone and $V(r)$ is the volume of the set of points that are a distance $r$ from $L$. Using the formula $f(r)=\cos \sqrt{a} r$, we obtain

$$
\begin{aligned}
\left(-\frac{\partial^{2}}{\partial r^{2}}-\frac{\partial}{\partial r}(\log V(r)) \frac{\partial}{\partial r}\right) \cos \sqrt{a} r & =a(\cos \sqrt{a} r)+\frac{\sqrt{a}(\sin \sqrt{a} r) V^{\prime}(r)}{V(r)} \\
& =a q f(r)=a q \cos \sqrt{a} r
\end{aligned}
$$

which implies that

$$
(q-1)(\cos \sqrt{a} r)=\frac{(\sin \sqrt{a} r) V^{\prime}(r)}{\sqrt{a} V(r)} .
$$

Using the fact that $\lim _{r \rightarrow 0} \frac{V(r)}{r^{q-1}} \operatorname{Vol}\left(S^{q-1}(1)\right) \operatorname{Vol}(L)$, part (5.1) follows.

Conversely, suppose that the four conditions hold. If $r$ is the distance from the leaf $L$ corresponding to one of the poles and $C(r)$ denotes the set of points in $M$ that are a distance $r$ from $L$, then the function $f(r)=\cos \sqrt{a} r$ satisfies

$$
\begin{aligned}
\frac{\int_{M} f \Delta_{B} f}{\int_{M} f^{2}} & =\frac{\int_{0}^{\pi / \sqrt{a}} f(r)\left(\int_{C(r)}(\Delta f) d V_{C(r)}\right) d r}{\int_{0}^{\pi / \sqrt{a}}(f(r))^{2} V(r) d r} \\
& =\frac{\int_{0}^{\pi / \sqrt{a}} f(r)\left(P_{r} \Delta f(r)\right) V(r) d r}{\int_{0}^{\pi / \sqrt{a}}(f(r))^{2} V(r) d r} \\
& =\frac{\int_{0}^{\pi / \sqrt{a}} f(r)\left(-\frac{\partial^{2}}{\partial r^{2}}-\frac{\partial}{\partial r}(\log V(r)) \frac{\partial}{\partial r}\right) f(r) V(r) d r}{\int_{0}^{\pi / \sqrt{a}}(f(r))^{2} V(r) d r} \\
& =a q .
\end{aligned}
$$

Since $f(r)=\cos \sqrt{a} r$ integrates to zero, the calculation above implies that $\lambda_{B} \leq a q$. Equality follows from the generalized Lichnerowicz inequality (Theorem 4.4).

\section{Examples.}

Example 6.1. Let $\Gamma$ be a discrete subgroup of $O(q)$, and let $S^{q}\left(\frac{1}{a}\right)$ be the $q$-sphere of radius $\frac{1}{a}$ in $\mathbb{R}^{q+1}$. Let $\Gamma$ act on $S^{q}\left(\frac{1}{a}\right) \subset \mathbb{R}^{q+1}$ by acting on the first $q$ coordinates by orthogonal transformations; this is a left action by isometries. Let $X$ be any Riemannian manifold of volume 1 such that there is a homomorphism $\pi_{1}\left(X, x_{0}\right) \stackrel{\phi}{\longrightarrow} \Gamma$. Let $\pi_{1}\left(X, x_{0}\right)$ act on the universal cover $\widetilde{X}$ on the right by deck transformations. We let $M$ be the suspension of $\phi$; that is, let $M=\widetilde{X} \times S^{q}\left(\frac{1}{a}\right) / \sim$, where $(\widetilde{x}[\gamma], y) \sim(\widetilde{x}, \phi([\gamma]) y)$ for every $[\gamma] \in \pi_{1}\left(X, x_{0}\right)$. The product-like metric is bundle-like for $(M, \mathcal{F})$, where the leaves of $\mathcal{F}$ are sets of $\sim$-equivalence classes of the form $\left\{\left(\widetilde{x}, y_{0}\right)_{\sim} \mid \widetilde{x} \in \tilde{X}\right\}$. 
The normal Ricci curvature of $(M, \mathcal{F})$ is precisely the Ricci curvature of $S^{q}\left(\frac{1}{a}\right)$ and thus satisfies

$$
\operatorname{Ric}^{\perp}(X, X)=a(n-1)|X|^{2}
$$

for every nonzero vector $X$ in $N \mathcal{F}$. The mean curvature of the foliation is zero, so the basic Laplacian is a restriction of the ordinary Laplacian on $M$. Thus, the basic Laplacian is a restriction of the Laplacian on the sphere $S^{q}\left(\frac{1}{a}\right)$. Also, the $(q+1)$-coordinate function $y^{q+1}$ is constant on the leaves and is an eigenfunction of the sphere's Laplacian with the lowest positive eigenvalue $a q$. Thus, $\lambda_{B}=a q$, and the foliation is transversally isometric to $S^{q}\left(\frac{1}{a}\right) / \operatorname{im}(\phi)$. This is an example of the setting of Theorem 5.1.

Observe that we may modify the metric along the leaves in the example above without changing the Ricci curvature bound. If we modify it so that the volume of each leaf does not change, we remain in the situation of Theorem 5.1; the basic Laplacian does not change as an operator on basic functions. However, if we modify the metric by multiplying the leafwise metric by a smooth function (whose average is not one), the basic Laplacian does change as an operator to

$$
\Delta_{B}^{\prime} f=\delta_{B}^{\prime} d f=\frac{1}{\psi} \delta_{B}(\psi d f),
$$

where $\psi$ is a positive, smooth, basic function, as in Proposition 4.1. Note that $\psi$ has no effect on the normal Ricci curvature. Applying this result to a simple foliation yields an interesting result about differential operators on spheres.

Example 6.2. Let $S^{q}\left(\frac{1}{a}\right)$ be the standard $q$-sphere of radius $\frac{1}{a}$ in $\mathbb{R}^{q+1}$. Let $X$ be any Riemannian manifold of volume 1 , and let $M=X \times S^{q}\left(\frac{1}{a}\right)$. The product metric is bundle-like for $(M, \mathcal{F})$, where the leaves of $\mathcal{F}$ are the sets of the form $L_{y_{0}}=\left\{\left(x, y_{0}\right) \mid x \in X\right\}$. The normal Ricci curvature of $(M, \mathcal{F})$ is the Ricci curvature of $S^{q}\left(\frac{1}{a}\right)$ and again satisfies

$$
\operatorname{Ric}^{\perp}(X, X)=a(q-1)|X|^{2}
$$

for every nonzero vector $X$ in $N \mathcal{F}$. The mean curvature of the foliation is zero, and basic functions may be thought of as functions on $S^{q}\left(\frac{1}{a}\right)$. Under this identification, the $L^{2}$ metrics on forms are preserved. Thus, the basic Laplacian is the Laplacian on the sphere $S^{q}\left(\frac{1}{a}\right)$. We now modify the leafwise metric so that the volume form on each $L_{y_{0}}$ is multiplied by the basic function $\psi$. The resulting basic Laplacian on functions is the operator

$$
\Delta_{\psi} f=\frac{1}{\psi} \delta(\psi d f)
$$


on $C^{\infty}\left(S^{q}\left(\frac{1}{a}\right)\right)$, which is self-adjoint with respect to the metric

$$
\langle f, g\rangle_{\psi}=\int_{S^{q}\left(\frac{1}{a}\right)} f(\sigma) g(\sigma) \psi(\sigma) d V(\sigma) .
$$

The generalized Lichnerowicz inequality states that the smallest positive eigenvalue $\lambda_{\psi}$ of $\Delta_{\psi}$ on $L^{2}\left(S^{q}\left(\frac{1}{a}\right)\right)$ satisfies

$$
\lambda_{\psi} \geq a q,
$$

with equality if and only if $\psi$ is a constant function.

\section{References}

[1] J.A. Álvarez-López, The basic component of the mean curvature of Riemannian foliations, Ann. Global Anal. Geom., 10 (1992), 179-194, MR 93h:53027, Zbl 0759.57017.

[2] I. Chavel, Eigenvalues in Riemannian Geometry, Orlando, Academic Press, 1984, MR 86g:58140, Zbl 0551.53001.

[3] S.Y. Cheng, Eigenvalue comparison theorems and its geometric applications, Math. Z., 143 (1975), 289-297, MR 51 \#14170, Zbl 0329.53035.

[4] D. Domínguez, Finiteness and tenseness theorems for Riemannian foliations, Amer. J. Math., 120(6) (1998), 1237-1276, MR 99k:53045, Zbl 0964.53019.

[5] J. Eells and L. Lemaire, Selected Topics in Harmonic Maps, Providence, Amer. Math. Soc. C. B. M. S., 50 (1983), MR 85g:58030, Zbl 0515.58011.

[6] J. Hebda, Curvature and focal points in Riemannian foliations, Indiana Univ. Math. J., 35(2) (1986), 321-331, MR 87g:53051, Zbl 0596.53027.

[7] F.W. Kamber and Ph. Tondeur, De Rham-Hodge theory for Riemannian foliations, Math. Ann., 277 (1987), 415-431, MR 89d:53070, Zbl 0637.53043.

[8] J. Lee and K. Richardson, Riemannian foliations and eigenvalue comparison, Ann. Global Anal. Geom., 16 (1998), 497-525, MR 99k:53047, Zbl 0915.53015.

[9] A. Lichnerowicz, Geometrie des Groupes des Transformationes, Travaux et Recherches Mathématiques, III, Dunod, Paris, 1958, MR 23 \#A1329, Zbl 0096.16001.

[10] P. Molino, Riemannian Foliations, Boston, Birkhäuser, 1988, MR 89b:53054, Zbl 0633.53001.

[11] S. Nishikawa, M. Ramachandran and $\mathrm{Ph}$. Tondeur, The heat equation for Riemannian foliations, Trans. Amer. Math. Soc., 319 (1990), 619-630, MR 90j:58145, Zbl 0702.58074.

[12] M. Obata, Certain conditions for a Riemannian manifold to be isometric with a sphere, J. Math. Soc. Japan, 14 (1962), 333-340, MR 25 \#5479, Zbl 0115.39302.

[13] E. Park and K. Richardson, The basic Laplacian of a Riemannian foliation, Amer. J. Math., 118 (1996), 1249-1275, MR 97i:58165, Zbl 0865.58047.

[14] W. Poor, Differential Geometric Structures, New York, McGraw-Hill, 1981, MR 83k:53002, Zbl 0493.53027.

[15] B. Reinhart, Differential Geometry of Foliations, Springer-Verlag, 1983, MR 85i:53038, Zbl 0506.53018. 
[16] H. Rummler, Quelques notions simples en géométrie et leur applications aux feuilletages compacts, Comment. Math. Helv., 54 (1979), 224-239, MR 80m:57021, Zbl 0409.57026.

[17] E. Salem, Riemannian foliations and pseudogroups of isometries, in 'Riemannian foliations' (ed. P. Molino), Boston, Birkhäuser, (1988), 265-296, MR 89b:53054, Zbl 0633.53001.

[18] Ph. Tondeur, Geometry of Foliations, Monographs in Mathematics, 90, Basel, Birkhäuser, 1997, MR 98d:53037, Zbl 0905.53002.

Received December 5, 2000.

Department of Mathematics

TEXAS TECH UNIVERSITY

LuBBOCK, TX 79409-2013

E-mail address: j-lee@math.ttu.edu

Department of Mathematics

Texas Christian University

Box 298900

FORT WorTh, TX 76129

E-mail address: k.richardson@tcu.edu 